\title{
Atuação do enfermeiro na prevenção das síndromes hipertensivas na gestação no âmbito da atenção básica
}

\author{
Nurse's performance in preventing hypertensive syndromes in pregnancy within the basis \\ of basic care
}
Rendimiento de la enfermera en la prevención de síndromes hipertensivos en el embarazo dentro de la base de cuidado básico

\begin{abstract}
Edivania Cristina da Silva ${ }^{1 *}$, Niedja Carla Dias de Lira e Silva ${ }^{2}$. Ada Evellyn Galdino da Silva ${ }^{3}$, Rayanne Lúcia de Oliveira Campos ${ }^{4}$, Manoela Rodrigues de Santana ${ }^{5}$, Luany Abade Café ${ }^{4}$, Paloma Maria Oliveira de Almeida ${ }^{6}$, Sandra Maria de Oliveira $^{3}$, Adriana dos Santos Gomes ${ }^{7}$, Adrian Thaís Cardoso Santos Gomes da Silva ${ }^{4}$.
\end{abstract}

\section{RESUMO}

Objetivo: Descrever a atuação do enfermeiro na prevenção das síndromes hipertensivas que acometem as mulheres durante o período gestacional, no âmbito da atenção básica. Revisão bibliográfica: O pré-natal é a ferramenta mais importante e completa para o acompanhamento da gestante, que tem como principal objetivo, assegurar o bem estar materno e fetal. O enfermeiro, juntamente com a equipe de estratégia saúde da família, representa um importante papel no rastreamento/diagnóstico, bem como no manejo das síndromes hipertensivas na gestação (SHEG), sendo essa patologia um problema de saúde pública, podendo causar danos tanto maternos quanto infantil. Considerações finais: Diante disso, é de suma importância a necessidade de buscar conhecimento acerca das SHEG, principalmente, para a equipe de enfermagem que possui um maior protagonismo no cenário da atenção básica. Munido de evidências científicas obtém-se uma padronização na assistência, por conseguinte será mais efetiva, rápida e eficaz. Propiciando cuidados às gestantes predispostas a desenvolver SHEG, garantindo uma melhor qualidade de vida durante e após a gestação e, consequentemente, para o neonato.

Palavras-chave: Complicações cardiovasculares na gravidez, Hipertensão, Enfermagem.

\begin{abstract}
Objective:To describe the role of nurses in the prevention of hypertensive syndromes that affect women during pregnancy, in the context of primary care. Bibliographic review: Prenatal care is the most important and complete tool for pregnant women's follow-up, whose main objective is to ensure maternal and fetal well being. The nurse, together with the family health strategy team, plays an important role in screening/diagnosis, as well as in the management of hypertensive syndromes during pregnancy (SHEG), and this pathology is a public health problem that can cause both maternal and infant damage. Final considerations: In front of this, it is of great importance the necessity to seek knowledge about the SHEG, mainly, for the nursing team that

${ }^{1}$ Centro Universitário dos Guararapes (UNIFG), Jaboatão dos Guararapes - PE. *E-mail: edicris027@gmail.com ${ }^{2}$ Centro Universitário São Miguel (Unisãomiguel), Recife - PE.

${ }^{3}$ Centro Universitário Estácio do Recife, Recife - PE.

${ }^{4}$ Universidade Federal de Pernambuco, Recife - PE.

${ }^{5}$ Universidade Católica de Pernambuco, Recife - PE.

${ }^{6}$ Faculdade Pernambucana de Saúde, Recife - PE.

${ }^{7}$ Centro de Referência em Saúde do Trabalhador (CEREST Regional Recife), Recife - PE.
\end{abstract}

SUBMETIDO EM: 1/2021

ACEITO EM: 1/2021

PUBLICADO EM: 2/2021 
has a bigger protagonism in the scene of the basic attention. Armed with scientific evidence it is obtained a standardization in the assistance, therefore it will be more effective, fast and efficient. Providing care to pregnant women predisposed to develop SHEG, ensuring a better quality of life during and after pregnancy and, consequently, for the newborn.

Keywords: Cardiovascular complications in pregnancy, Hypertension, Nursing.

\section{RESUMEN}

Objetivo: Describir el papel de las enfermeras en la prevención de los síndromes de hipertensión que afectan a las mujeres durante el embarazo, en el ámbito de la atención básica. Revisión bibliográfica: La atención prenatal es la herramienta más importante y completa para el seguimiento de las mujeres embarazadas, cuyo principal objetivo es asegurar el bienestar materno y fetal. I La enfermera, junto con el equipo de estrategia de salud familiar, desempeña un papel importante en el cribado/diagnóstico, así como en el manejo de los síndromes de hipertensión en el embarazo (SHEG), y esta patología es un problema de salud pública, que puede causar daños tanto a la madre como al niño. Consideraciones finales: Frente a esto, es de gran importancia la necesidad de buscar el conocimiento sobre el SHEG, principalmente, para el equipo de enfermería que tiene un mayor protagonismo en el escenario de la atención básica. Armado con pruebas científicas, se obtiene una estandarización en el cuidado, por lo que será más efectivo, rápido y eficiente. Proporcionar cuidados a las mujeres embarazadas predispuestas a desarrollar SHEG, asegurando una mejor calidad de vida durante y después del embarazo y, por consiguiente, al recién nacido.

Palabras clave: Complicaciones cardiovasculares del embarazo, Hipertensión, Enfermería.

\section{INTRODUÇÃO}

A Atenção Básica $(A B)$ ao longo dos anos, vem se tornando mais complexa e resolutiva. Com isso, a exigência de enfermeiros qualificados para atender às demandas dessa complexidade tornaram-se cada vez maiores. O cuidado prestado ao ser humano perpassa todas as etapas da vida. Na mulher, em especial destacamos a gestação, que é um período fisiológico e complexo para a gestante. O qual vem acompanhado de inúmeras mudanças, dentre as quais, podemos ressaltar as corporais, hormonais e psicológicas. A mulher tem como porta de entrada a AB no Sistema Único de Saúde (SUS). Na Unidade Básica de Saúde (UBS), são fornecidos todos os recursos necessários para o acompanhamento de uma gestação segura e saudável (MARIANO MSB, et al., 2018; LIMA JP, et al., 2018).

Diante de um conjunto de serviços oferecidos na UBS, o pré-natal é a ferramenta mais importante e completa para o acompanhamento da gestante, que tem como principal objetivo, assegurar o bem estar materno e fetal. O pré-natal deve contar com no mínimo seis consultas, pautadas no atendimento holístico, humanizado e individualizado, que devem ser realizadas pelo enfermeiro ou médico, de maneira intercaladas e associadas a uma abordagem interdisciplinar. Durante esse atendimento continuado à gestante, é possível realizar a prevenção e detecção precoce de patologias que possam atrapalhar o andamento fisiológico da gestação, parto e pós-parto. Além disso, a gestante realizará consultas com o odontólogo, nutricionista e outros profissionais da equipe, de acordo com suas demandas de saúde (LIMA JP, et al., 2018).

Dentre as complicações que as gestantes podem desenvolver no ciclo gravídico-puerperal, de maneira mais comum, encontra-se a Síndrome Hipertensiva Específica da Gestação (SHEG), patologia que atualmente é a maior responsável em desfechos de mortes perinatais. Tal patologia é caracterizada por manifestações clínicas como a Hipertensão Arterial Sistêmica (HAS) após a vigésima semana de gestação, podendo ter associado a presença de proteinúria, sendo assim denominada como Pré-eclâmpsia. Em casos mais graves, a gestante pode apresentar quadros convulsivos associados aos relatados anteriormente, caracterizando a Eclampsia (MAGNAGO C e PIERANTONI CR, 2020; OLIVEIRA GS, et al., 2017).

O diagnóstico da Hipertensão Gestacional é dado quando os níveis pressóricos são iguais ou superiores a 140/90 mmHg, após a vigésima semana de gestação. É importante ressaltar que, a resolução definitiva da 
SHEG é a interrupção da gestação, que poderá ocorrer através da indução do parto vaginal ou através da cesariana. A HELLP Síndrome (Haemolysis, Elevated Liver enzyme activity e Low Platelets) ou Coagulação Intravascular Disseminada (CID), é provocada pelo quadro da SHEG, que se caracteriza de maior gravidade, o qual necessita de cuidados intensivos e de forma ágil para a preservação da vida materna e fetal (THULER ACMC, et al., 2018).

No pré-natal o acompanhamento adequado proporciona a prevenção e a detecção precoce de SHEG, reduzindo as taxas de morbimortalidade devido a complicações relacionadas a essa patologia. As gestantes que forem identificadas com hipertensão deverão ser referenciadas para a realização do pré-natal em serviço especializado de alto risco de acordo com as recomendações do Ministério da Saúde. Aquelas que forem identificadas com quadros de pré-eclâmpsia ou eclampsia, deverão ser encaminhadas imediatamente aos serviços de urgências e emergências obstétricas (LIMA JP, et al., 2018).

Tendo em vista a alta incidência de gestantes que apresentam a Síndrome Hipertensiva e que a detecção precoce se relaciona à consulta de pré-natal, o presente estudo objetivou descrever a atuação do enfermeiro na prevenção das síndromes hipertensivas que acometem as mulheres durante o período gestacional, no âmbito da atenção básica e as consequências dessas ações na sua saúde e durante o ciclo gravídicopuerperal e do feto.

\section{REVISÃO BIBLIOGRÁFICA}

\section{Atenção básica e pré-natal}

A saúde brasileira passou por diversos marcos e contextos históricos que culminaram na criação e mudanças da Atenção Básica para que ela seja como é atualmente. A saúde pública no século XX era pautada na doença e em ações campanhistas, de controle de endemias e epidemias, em seguida obtiveram conquistas no que diz respeito a criação de órgãos públicos que assumiram a responsabilidade de garantir ações que promovessem a saúde da população brasileira. Contudo, o contexto de saúde populacional não era dos melhores, o que ocasionava muita insatisfação por parte da população (NETO DC, 2017).

Nesse contexto, o Movimento da Reforma Sanitária Brasileira ganhou força após a Conferência de Alma Ata, suas ações e iniciativas culminaram na criação do Sistema Único de Saúde, por meio da Constituição Federal de 1988. Em 1994, o Ministério da Saúde lançou o Programa de Saúde da Família (PSF), que foi se consolidando aos poucos, foram realizadas melhorias e passou a ser chamado como Atenção Primária (NETO DC, 2017).

A Organização Mundial da Saúde (OMS), Organização Pan-Americana da Saúde (OPAS) e os governos, acreditam na produção da transformação e regulação do sistema de Atenção à Saúde, com o acesso universal e de proteção social à saúde. A produção de respostas às necessidades e expectativas em relação ao risco e doença, promoção de comportamentos e estilo de vida saudáveis e a prevenção de danos sociais e ambientais sobre a saúde, fazem parte do processo de reestruturação, fortalecimento e racionalização dos sistemas públicos de saúde, esses fatores destacam-se como protagonistas essenciais para a atenção primária à saúde, que é considerada como o alicerce dos cuidados oferecidos pela ampla rede de serviços básicos do SUS (CECÍLIO LCO e REIS AAC, 2018).

A Atenção Primária à Saúde (APS) que é o primeiro nível de atendimento, desempenha uma forte influência sobre o acolhimento à gestante no serviço de saúde, as ações de promoção, proteção e recuperação de saúde integral e o desenvolvimento de ações continuadas, possibilitam a criação de vínculos entre profissionais de saúde e famílias. O vínculo com a comunidade, contribui para um entendimento global sobre o processo saúde-doença, os fatores determinantes e condicionantes que afetam a saúde da usuária, permitindo o desenvolvimento de intervenções que se sobrepõem ao desenvolvimento de práticas curativas e englobam a visão holística da mulher (THULER ACMC, et al., 2018).

Além disso, o Ministério da Saúde, com o intuito de dar continuidade na classificação elaborada em 1996 pela OMS implantou por meio do Programa de Humanização do Pré-Natal e Nascimento (PHPN), uma extensão do processo de humanização na obstetrícia. Essa estratégia tinha como propósito o resgate da 
atenção obstétrica qualificada, integrada e humanizada no pré-natal, parto e puerpério, com o envolvimento dos estados e municípios (SANTOS HFL e ARAÚJO MM, 2016). Um bom acesso, ter cobertura e acompanhamento pré-natal, a assistência ao parto e puerpério às gestantes e ao recém-nascido, na perspectiva dos direitos de cidadania, fundamentando-se no direito à humanização da assistência à parturiente e ao neonato como condição para acompanhamento do parto e do puerpério, são garantidos pelo PHPN (BRASIL, 2015).

Acesso ao atendimento dificultado, prevalência do início das consultas após 12 semanas de gestação, a não realização dos procedimentos, exames laboratoriais e de imagem insuficientes, carência na educação em saúde, baseada no acesso para a gestante a informações sobre seus direitos e os direitos de seus familiares, esses dados demonstram que o cuidado efetivo fica prejudicado, gerando resultados insatisfatórios e negativos refletindo diretamente no processo de saúde e doença da gestante, são falhas na atenção pré-natal (SEHNEM GD, et al., 2020).

Uma das grandes prioridades da saúde pública tem sido ações voltadas para a atenção materno-infantil. Destacando assim, a importância da assistência da mulher durante o ciclo gravídico puerperal, que compreende o pré-natal, parto e puerpério, essas ações apresentam uma redução atenuada nos índices de mortalidade materna e neonatal. Mortes maternas decorrentes de patologias como síndromes hipertensivas, hemorragias, doenças puerperais e doenças circulatórias, estão apresentando uma diminuição acentuada, no entanto a taxa de mortalidade por causas evitáveis, ainda é um dado preocupante (PEREIRA RMS, et al., 2019).

Para identificação das situações de saúde e doença com conhecimento científico, prescrevendo e implementando medidas de enfermagem que colaborem com a promoção, prevenção de agravos e doenças e o alívio do sofrimento, proteção da saúde, recuperação e reabilitação do indivíduo, proporciona cuidado ao paciente, família e comunidade, são utilizados na consulta de enfermagem princípios fundamentais da universalidade, equidade, resolutividade e integralidade das ações de saúde, reafirmando o respeito aos direitos humanos no exercício da profissão, em diferentes contextos socioambientais, culturais, étnicos, identidade de gêneros ou orientação sexual (COFEN, 2017).

Nesse pressuposto, a consulta de enfermagem no pré-natal possui um papel muito importante para diminuição das taxas de morbimortalidade materna e neonatal, visto que o acompanhamento no período gestacional e no pós-parto, o acolhimento e atendimento de qualidade, acompanhamento multidisciplinar dos usuários por profissionais qualificados, a resolutividade da assistência, abordagem coletiva e familiar, vacinação e a garantia da realização dos exames e rastreios necessários para a condição da gestante, fomentaram um atendimento individualizado, humanizado de abordagem holística que possibilitará a promoção e prevenção a saúde e detecção precoce de agravos e doenças que poderiam ter um desfecho negativo se não fossem previamente diagnosticados. Além disso, é importante que o enfermeiro durante a assistência elucide as dúvidas e oriente a gestante quanto aos seus direitos (CRUZ AL, 2019).

\section{Síndromes hipertensivas nas gestações}

Mesmo sendo desconhecida a etiologia da SHEG, acredita-se que alguns fatores sejam os responsáveis pelo desenvolvimento SHEG, tais como predisposições genéticas, falha no desenvolvimento da placenta, aspectos imunológicos, anormalidades na coagulação sanguínea, má adaptação circulatória, aumento na produção e relação do tromboxano A2/PGI2 (prostaciclina), dentre outros. Tais fatores acarretam uma redução na pressão de perfusão uteroplacentária, gerando hipóxia/isquemia da placenta durante a gestação (ABRAHÃO ACM, et al., 2010). Obesidade, raça negra, colagenose, idade materna $>40$ anos são fatores de risco de menor proporção e entre os fatores de risco de maior proporção estão: primigestação, gemelaridade, nova maternidade, hipertensão crônica, história prévia ou familiar (irmã ou mãe), diabetes, Síndrome do Anticorpo Antifosfolípide (SAAF).Outro fator que também pode interferir é o socioeconômico, que está interligado com renda, moradia, alimentação, informação e conhecimento sobre a gestação e as mudanças gestacionais. Estudos ainda mostram que continua sendo um grande desafio, a prevenção e o reconhecimento precoce da doença hipertensiva na gravidez (GONÇALVES ACO e THEODOROPOULOS TAD, 2020). 
A hipertensão crônica, na qual a gestante já previamente hipertensa, e continuou apresentando valores pressóricos alterados após doze semanas pós-parto; hipertensão gestacional que ocorre após a $20^{\circ}$ semana de gestação, não apresenta complicações como a proteinúria e volta aos seus valores pressóricos normais nas primeiras 12 semanas de puerpério; pré-eclâmpsia que consiste na junção da hipertensão com a proteinúria (300mg ou mais de proteína em urina de $24 \mathrm{~h}$ ) após a $20^{\circ}$ semana de gestação, em gestantes que anteriormente mantinham valores pressóricos normais são as síndromes hipertensivas (ANTUNES MB, et al., 2017).

Umas das patologias que mais acometem a mulher durante a gestação, é a SHEG, uma síndrome obstétrica que surge após a vigésima semana de gestação. É caracterizada pela tríade: hipertensão arterial, edema e/ou proteinúria, podendo culminar com convulsões e coma, geralmente aparece no terceiro trimestre gestacional, podendo estender-se até o puerpério, em cerca de 6 a 12 semanas após o parto (REZENDE JF e MONTENEGRO CAB, 2013).

A hipertensão gestacional pode permanecer após uma retirada do feto ou iniciar no puerpério, devido a esta condição é de suma importância o acompanhamento domiciliar pelo enfermeiro e a equipe da unidade de saúde da família, monitorando a pressão arterial e os demais sinais vitais, até que a mulher não apresente as complicações do quadro. Quando ocorre um acompanhamento de qualidade no pré-natal, na qual a SHEG é diagnosticada precocemente, pode-se reduzir a morbimortalidade na gestante e recém-nascido (ANTUNES MB, et al., 2017).

Para o rastreamento da pré-eclâmpsia em gestantes de alto risco, inclui-se a realização de doppler de artérias uterinas com 24 semanas, como resultados podem aparecer um fluxo de alta resistência, com incisuras protodiastólicas persistentes além de 23 semanas e/ou índice de pulsatilidade alterado (acima do percentil 95 para esta idade gestacional), estes resultados, por sua vez, identificam um alto risco para o desenvolvimento da pré-eclâmpsia. Entretanto, quando os exames estão normais após a 25 semanas, há uma grande chance do não acometimento por esta comorbidade nas gestantes de alto risco (GONÇALVES ACO e THEODOROPOULOS TAD, 2020).

O fluxo de atendimento preconizado pelo Ministério da Saúde descreve que todas as gestantes diagnosticadas com pré-eclâmpsia deverão ser referenciadas para a urgência obstétrica e, posteriormente, deverão ser acompanhadas pela atenção especializada. As gestantes diagnosticadas com hipertensão crônica ou que possuíam hipertensão prévia à gestação, também precisarão ser acompanhadas na atenção especializada (BRASIL, 2010).

As alterações hepáticas, cerebrais sanguíneas, útero placentárias, hidroeletrolíticas e o prognósticos de crises convulsivas, são alterações que afetam os sistemas vitais da mulher com síndrome hipertensiva gestacional, podendo levar gestante a óbito (OLIVEIRA GS, et al., 2017).

A gestação nas mulheres com pré-eclâmpsia, deve ser interrompida através de uma cesariana ou com a indução do trabalho de parto, tendo como possibilidade o risco de uma complicação como uma hemorragia, infecção e picos hipertensivos. Nesse contexto, é possível identificar o quanto é importante o cuidado no acompanhamento do pré-natal e no cuidado à gestante hipertensiva, no qual o enfermeiro é capaz de direcionar a assistência, melhorando a qualidade do atendimento e apresentam maior risco de síndromes metabólicas, doenças cardiovasculares e hipertensão sistêmica precoce, nas crianças de gestações acometidas de pré-eclâmpsia. Pacientes com pré-eclâmpsia pode apresentar em evolução da patologia muito diferentes, umas se estabilizam até o fim da gestação, outras tem a situação agravada ao longo de semanas, e algumas apresentam sinais de gravidade em dias ou até mesmo em horas (SARMENTO RS, et al., 2020).

\section{Atuação do enfermeiro}

O enfermeiro deverá possuir competência e formação suficientes para praticar seus conhecimentos de cunho técnico científico na prática assistencial, com um cuidado coerente, holístico e humanizado. Desta forma, a Sistematização da Assistência em Enfermagem (SAE), se torna uma atribuição específica e fundamental do enfermeiro no planejamento dos cuidados para a gestante, diferenciando-o dos demais profissionais da equipe multidisciplinar (ABRAHÃO ACM, et al., 2020). 
A enfermagem com seus cuidados específicos prestando atendimento a mulheres com pré-eclâmpsia e/ou eclampsia é capaz de reduzir complicações e taxas de morbimortalidade. Compreendendo um exame físico criterioso; identificando precocemente os sinais de pré-eclâmpsia/eclampsia; acompanhamento de exames laboratoriais; avaliação fetal; treinamentos dos profissionais, incluindo a necessidade de educação continuada; padronização do atendimento a partir de instrumentos; aferição da pressão arterial com manguito adequado à circunferência do braço; velocidade lenta de desinsuflação; necessidade de estabelecer a técnica de aferição da PA; identificação e tratamento precoce da crise hipertensiva mediante protocolos e normas vigente (SARMENTO RS, et al., 2020).

Para proteger a saúde do binômio mãe-filho a enfermagem tem um papel indispensável em todas as etapas do cuidado, desde o acolhimento à gestante quando é construído um vínculo, acompanhamento do processo do parto ao puerpério, prevenindo complicações e intercorrências, prestando cuidados imediatos e mediatos ao bebê após o nascimento, realizando o registro e refletindo sobre a assistência prestada com o intuito da preservação da vida humana (OLIVEIRA GS, et al., 2017). O encaminhamento desnecessário de gestantes para esses serviços pode ocasionar no congestionamento e falta de disponibilidade de vagas no sistema do SUS, porém, as gestantes com os critérios para o pré-natal de alto risco enfrentam longa espera para ter acesso a consulta e continuam o seu pré-natal na atenção primária, na qual o enfermeiro orienta adoção de hábitos e rotinas saudáveis para melhorar a qualidade de vida dessas gestantes, que posteriormente deverão fazer o acompanhamento conjunto da atenção primária em saúde com o pré-natal de alto risco (BRASIL, 2010).

O enfermeiro da Atenção Primária de Saúde, realiza a consulta de enfermagem, fazendo uma avaliação dinâmica das situações de risco identificando problemas como as síndromes hipertensivas durante a gestação, de forma a intervir no problema encontrado, de maneira a impedir um desfecho negativo. A realização do pré-natal de qualidade atua de forma positiva no resultado perinatal e na diminuição das taxas de mortalidade materna, ou seja, com intuito de assegurar um parto sem intercorrências, minimizando possíveis danos à saúde da mãe e do neonato (BRASIL, 2010).

Um enfermeiro ao conduzir o pré-natal, identifica e acompanha as mulheres que possuem fatores de risco para desenvolver síndromes hipertensivas, devendo ser investigado o histórico pessoal, gestacional e familiar como forma de reconhecer a predisposição da mulher desenvolver esses distúrbios (SILVA SN, et al., 2017). Considerando o papel como educador, sobretudo na atenção primária, o enfermeiro realiza orientações sobre o estilo de vida ideal para a gestante, com base na individualidade e atendimento humanizado, para que assim sejam capazes de promover a prevenção a saúde. Alguns pontos são essenciais para educação em saúde quanto a SHEG: orientar uma alimentação saudável com a finalidade de prevenir a obesidade, estimular a realização de exercícios leves como caminhadas, mudanças de comportamento como abandonar o tabagismo, visto que é considerado um fator de risco independente para a pré-eclâmpsia (THULER ACMC, et al., 2018).

Além disso, a mulher que desenvolveu doença hipertensiva também deve ser acompanhada no puerpério por meio de consulta de enfermagem e visita domiciliar, partindo do pressuposto que o problema poderá perdurar durante esse período. Sendo assim, é essencial que seja criado um plano de cuidados individualizado considerando os aspectos biopsicossociais, e visando a promoção do cuidado holístico (OLIVEIRA LAM, et al., 2018). Com um cuidado padrão ouro, realizado de acordo com os protocolos do Ministério da Saúde, a assistência da enfermagem consegue ter uma abordagem certa e humanizada, tendo como foco principal a gestante, obtendo assim um melhor resultado; maior capacidade no atendimento, diminuição dos gastos, menor tempo de internação pós-parto, redução da morbimortalidade materno-fetal, instituindo confiança e segurança as instituições de saúde (SARMENTO RS, et al., 2020).

\section{CONSIDERAÇÕES FINAIS}

A Estratégia Saúde da Família (ESF) vem mostrando a cada dia, sua importância enquanto principal forma de acesso para os usuários do SUS. O enfermeiro detém um papel de grande importância, pois tem a finalidade de prevenir e promover cuidados às mulheres que planejam uma gravidez ou estão gestantes, 
conscientizando-as acerca do pré-natal de forma correta e segura. Comumente, os profissionais se depararam com problemas de saúde que acometem gestantes, sendo uma das principais, a síndrome hipertensiva, que além de acarretar danos para a gestante e seu filho, representa um problema de saúde pública. Frente a esse exposto, o conhecimento científico referente às SHEG é de suma importância aos profissionais de enfermagem visto que, esse vai padronizar condutas que ajudam no raciocínio clínico e nas tomadas de decisões no atendimento da gestante auxiliando assim, na prestação de uma assistência mais rápida e eficaz. Por conseguinte, determinará novos cuidados às gestantes propensas a desenvolver SHEG, promovendo uma gestação mais saudável e contribuindo para uma melhor qualidade de vida da gestante e por conseguinte ao neonato.

\section{REFERÊNCIAS}

1. ABRAHÃO ACM, et al. Atuação do Enfermeiro a Pacientes Portadoras de Síndrome Hipertensiva Específica na Gestação. Rev Cient Esc Estadual Saúde Pública Goiás "Cândido Santiado", 2020; 6(1): 51-56.

2. ANTUNES MB, et al. Síndrome hipertensiva e resultados perinatais em gestação de alto risco. Rev Min Enferm, 2017; 15.

3. BRASIL, Ministério da Saúde. Departamento de Ações Programáticas Estratégicas. Gestação de alto risco: manual técnico. Ministério da Saúde, 2010; 1(5): 302-305.

4. BRASIL, Ministério da Saúde. Cadernos HumanizaSUS. [s.I.: s.n.], 2015.

5. CECILIO LCO, REIS AAC. Notes on persistent challenges for basic health care in Brazil. Cadernos de Saúde Pública,2018; 34(8): 1-14.

6. COFEN, Conselho Federal de Enfermagem. Resolução 564/2017 dispõe sobre o Código de Deontologia de Enfermagem, 2017.

7. CRUZ AL. Importância da Atenção Pré-natal na prevenção de complicações na gestação: revisão integrativa. Dissertação (Graduação em Enfermagem) - Universidade Católica do Salvador, Bahia, 2019.

8. FERNANDES JA, et al. Avaliação da atenção à gestação de alto risco em quatro metrópoles brasileiras. Cad. Saúde Pública, 2020; 36(5).

9. GENNARO S, et al. Improving prenatal care for minority women. Am J Matern Child Nurs, 2016; 41(3): 147-53.

10. GONÇALVES ACO, THEODOROPOULOS TAD. Manejo das Doenças Hipertensivas Gestacionais - Revisão de Diagnóstico, Tratamento e Prevenção. Revista Corpus Hippocratium, 2020;1 (1)3.

11. LIMA JP, et al. Perfil socioeconômico e clínico de gestantes com Síndrome Hipertensiva Gestacional. Rev.rene, 2018; 19(1): 3455.

12. MAGNAGO C, PIERANTONI CR. A formação de enfermeiros e sua aproximação com os pressupostos das Diretrizes Curriculares Nacionais e da Atenção Básica. Ciênc. saúde coletiva, 2020; 25(1): 15-24.

13. MARIANO MSB, et al. Mulheres com síndromes hipertensivas. Rev Enferm UFPE on line, 2018;12(6):1618-24.

14. NETO DC. A (Des)medicalização na Atenção Primária: o surgimento de um novo cenário na saúde pública. Revista Científica Fagoc Saúde, 2017: 1(2): 9-14.

15. OLIVEIRA GS, et al. Assistência de enfermeiros na síndrome hipertensiva gestacional em hospital de baixo risco obstétrico. Rev Cuid, 2017; 8(2): 1561-72.

16. OLIVEIRA LAM, et al. Cuidados de enfermagem à gestante com síndrome hipertensiva: revisão integrativa. Brazilian $\mathrm{J}$ of Surgery and Clinical Research-BJSCR, 2018; 23(2): 159-164.

17. PEREIRA RMS, et al. Conhecimentos, atitudes e prática de enfermeiras frente à gestante com hipertensão. Revista uningá, 2019; 56:157-168.

18. REZENDE J, MONTENEGRO CAB. Obstetrícia Fundamental. Guanabara Koogan, 2013.

19. SANTOS HFL, ARAÚJO MM. Políticas De Humanização Ao Pré-Natal E Parto: Uma Revisão De Literatura Humanization the Policies Prenatal and Childbirth: a Literature Review. Revista Científica FacMais, 2016; 6(2): 5464.

20. SARMENTO RS, et al. Pré-eclâmpsia na Gestação: Ênfase na Assistência de Enfermagem. Enfermagem Brasil, 2020;19(3):261-267.

21. SEHNEM GD, et al. Consulta de pré-natal na atenção primária à saúde: fragilidades e potencialidades da intervenção de enfermeiros brasileiros. Revista de Enfermagem Referência, 2019; 5(1): 19050.

22. SILVA SN, et al. A importância do Pré-natal na Prevenção da Toxemia Gravídica e o Papel do Enfermeiro. Rev Saúde Foco, 2017; 9: 16.

23. THULER ACMC, et al. Medidas Preventivas das Síndromes Hipertensivas da Gravidez na Atenção Primária. Rev. Enfer. UFPE online, 2018: 1060-1071. 